\title{
A Case of Lemierre's Syndrome in Intensive Care Unit
}

\author{
Man-Yee Man, Hoi-Ping Shum, Wing-Wa Yan, Susanna K. P. Lau ${ }^{1}$ \\ Department of Intensive Care, Pamela Youde Nethersole Eastern Hospital, ${ }^{1}$ Department of Microbiology, The University of Hong Kong, Hong Kong SAR, China
}

\section{Abstract}

We report a 21-year-old young male with Lemierre's syndrome presented as tonsillitis and Fusobacterium septicemia with respiratory failure and required intensive care. Lemierre's syndrome is the septic embolic complication of recent pharyngeal illness. Fusobacterium spp. accounts for the majority of cases. High index of suspicion is needed and prolonged antibiotic is advised. It is seldom seen in intensive care but should never be forgotten.

Keywords: Critical care, Fusobacterium necrophorum, Lemierre's syndrome

\section{INTRODUCTION}

Lemierre's syndrome is the septic embolic complication of recent pharyngeal illness. It is commonly caused by Fusobacterium spp. We present a case of young man with Lemierre's syndrome and provide literature review on management principles.

\section{Case Report}

A 21-year-old male with unremarkable past health except was allergic to cefuroxime and metronidazole presented with sore throat and shortness of breath. He was initially seen by otorhinolaryngologist for left tonsillitis in the Emergency Department. Flexible laryngoscopy showed mobile bilateral vocal cords with patent upper airway. He was in septic shock and transferred to the Intensive Care Unit for acute tonsillitis complicated with septic shock on October 7, 2016.

Detailed medical history reviewed a 4-day history of sore throat, fever, and poor oral intake. He was febrile with low-grade temperature of $37.6^{\circ} \mathrm{C}$. Chest X-ray showed bilateral lower zone infiltrates. Ultrasound thorax showed bilateral pleural effusion around $4 \mathrm{~cm}$ without septation. He developed respiratory failure and intubated for respiratory support.

He was treated with doxycycline, clindamycin, and vancomycin empirically in view of the drug allergy history. Upon clinical deterioration, antibiotics were upgraded to meropenem and doxycycline.

\begin{tabular}{|l|l|}
\hline \multicolumn{3}{|c|}{ Access this article online } \\
\hline Quick Response Code: & Website: \\
& www.ijccm.org \\
& \\
\end{tabular}

Throat swab, urine, and sputum culture were negative. Endotracheal aspirates for influenza and other viruses were negative and bacterial culture was negative. Blood culture on day 2 yielded Fusobacterium necrophorum, sensitive to metronidazole and minimal inhibitory concentration of clindamycin $0.12 \mu \mathrm{g} / \mathrm{mL}$. Microbiologists were consulted and suggested to keep meropenem alone.

In view of persistent leukocytosis and neck pain, computed tomography $(\mathrm{CT})$ of neck and thorax was performed on October 13, 2016, which showed no obvious abscess, but left internal jugular thrombophlebitis [Figure 1] and multiple cavitating lung nodules [Figure 2] compatible with distal thromboembolism and supported the diagnosis of Lemierre's syndrome. Right internal jugular vein, left brachiocephalic vein, and superior vena cava were patent. Otorhinolaryngologist suggested to continue antibiotics and not for internal jugular vein ligation. He was shortly discharged to general ward to continue intravenous amoxicillin-clavulanate following the antibiotic desensitization protocol. He made satisfactory progress and was discharged home with oral amoxicillin-clavulanate. No anticoagulation was given. Subsequent follow-up with chest X-ray showed resolution of lung infiltrates, and antibiotic was stopped after 6 weeks.

Address for correspondence: Dr. Man-Yee Man, Department of Intensive Care, Pamela Youde Nethersole Eastern Hospital, 3 Lok Man Road, Chai Wan, Hong Kong SAR, China. E-mail: mmy553@ha.org.hk

This is an open access article distributed under the terms of the Creative Commons Attribution-NonCommercial-ShareAlike 3.0 License, which allows others to remix, tweak, and build upon the work non-commercially, as long as the author is credited and the new creations are licensed under the identical terms.

For reprints contact: reprints@medknow.com

How to cite this article: Man MY, Shum HP, Yan WW, Lau SK. A case of Lemierre's syndrome in Intensive Care Unit. Indian J Crit Care Med 2018;22:122-4. 


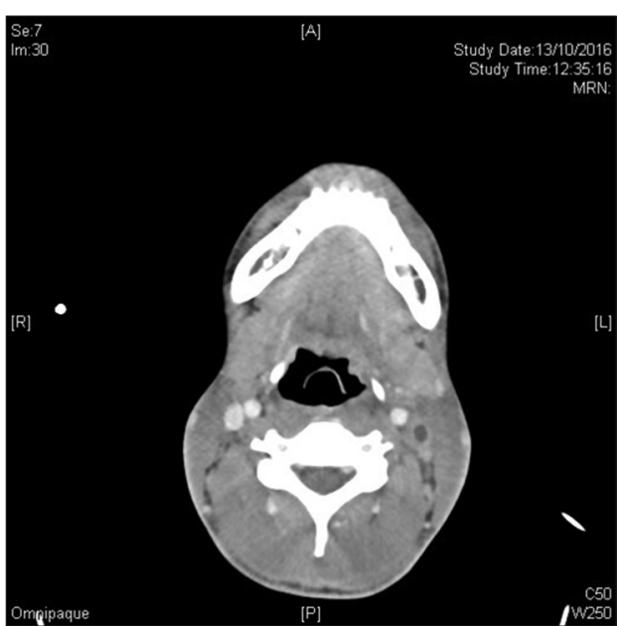

Figure 1: Thrombosis of left internal jugular vein and its branches on contrast computed tomography

\section{Discussions}

Lemierre's syndrome is the septic thrombophlebitis following an oropharyngeal infection. ${ }^{[1]}$ It was first described by Lemierre et al. in 1936 . Up to $60 \%$ of cases were caused $F$. necrophorum infection. ${ }^{[2]}$ In a systematic review by Johannesen and Bodtger, other pathogens causing Lemierre's syndrome include Streptococcus, Staphylococcus aureus, Klebsiella spp., and Pseudomonas. ${ }^{[3]}$ Septic emboli in the liver and lung were commonly identified, ${ }^{[4]}$ but rarer complications including necrotizing fasciitis, mycotic aneurysms, and central nervous system infections have also been reported. ${ }^{[3,5,6]}$ Lemierre's syndrome, often known as "the forgotten disease," has a markedly reduced incidence since the 1940 s, probably due to the widespread use of antimicrobials.

The disease tends to affect young adults who were otherwise healthy. ${ }^{[1,7]}$ Men are two times more likely to be affected than women. More than two-third of the patients presented with tonsillitis, pharyngitis, or upper respiratory tract infections. ${ }^{[1]}$ Increased incidence during late winter has been observed. ${ }^{[7]}$

Patients suffering from Lemierre's syndrome infrequently require invasive organ supports. In our patient's case, he presented with septic shock and shortly intubated for mechanical ventilation. Prognosis is generally good with low mortality, but recurrence of abscess can sometimes happen.

F. necrophorum is an obligate Gram-negative anaerobic bacterium. It is commonly found in oral cavity, and up to $20 \%$ of healthy teenagers are colonized with F. necrophorum. It may be the second most common bacterial cause of pharyngotonsillitis of adolescents. ${ }^{[7]}$ Coinfection with virus and bacteria, i.e., Streptococcus has been reported. ${ }^{[7]}$ Simultaneous Epstein-Barr virus infection was reported in up to $19 \%$ of cases. $^{[7]}$

F. necrophorum is difficult to culture and requires a longer incubation period. Identification of $F$. necrophorum to species

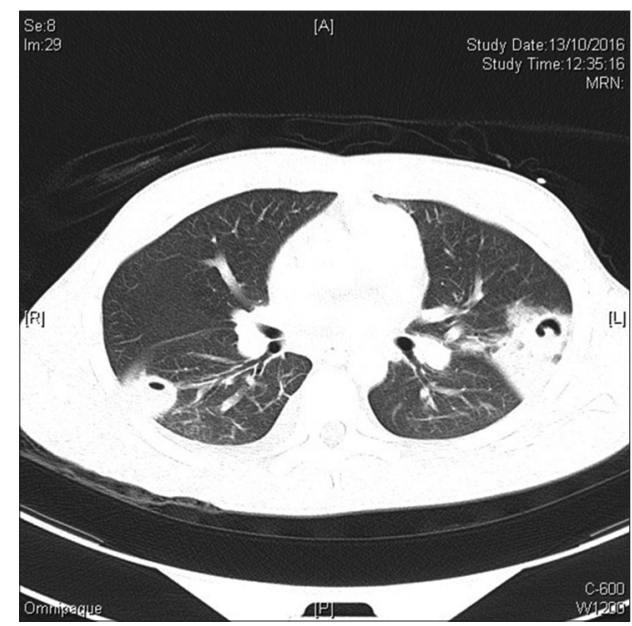

Figure 2: Computed tomography thorax shows multiple cavitary lung lesions

level often relies on newer microbiological techniques, i.e., $16 \mathrm{~s}$ ribosomal RNA and matrix-assisted laser desorption/ionization time-of-flight. ${ }^{[7]}$ In our patient, prolonged incubation of the blood culture yielded F. necrophorum.

Different imaging modalities assist in the diagnosis of Lemierre's syndrome. Doppler ultrasound of the internal jugular veins allows a quick screening for thrombophlebitis and is especially helpful as a bedside tool in intensive care settings. Moreover, central venous cannulations in such affected vein should be avoided. CT scan confirms and delineates the extent of thrombophlebitis and also allows the identification of lung abscess in our patient. In fact, more than $95 \%$ of cases of internal jugular thrombophlebitis were diagnosed by $\mathrm{CT}^{\left[{ }^{[3]}\right.}$ Serial scans also allow disease monitoring and possibly guide antibiotics and anticoagulation treatments.

Management of Lemierre's syndrome lies on early adequate and prolonged course of antibiotics. The debate for anticoagulation is ongoing, yet it may seem reasonable to anticoagulate high-risk patients. Surgical interventions including abscess drainage may be helpful, but venous ligation is seldom indicated.

Varieties of treatment durations have been suggested. In general, a prolonged course of antibiotics around 3-6 weeks is generally recommended. Metronidazole is often the recommended agent with adequate tissue penetration. ${ }^{[1]}$ A combination of third-generation cephalosporin and metronidazole would provide a reasonable coverage for other coinfections with Streptococcus. ${ }^{[2]}$ Erythromycin resistance is not uncommon and up to $20 \%$ has been reported. Antibiotics can be initially given intravenously for 2-3 weeks then orally up to 6 weeks. ${ }^{[8]}$

The role, duration, and targets of anticoagulation were uncertain. A retrospective analysis of 18 cases with or without anticoagulation showed no difference in thrombi resolution, ${ }^{[9]}$ while another retrospective review of pediatric patients did not show major bleeding complications on anticoagulation. ${ }^{[10]} \mathrm{A}$ systematic review of 137 cases found that more than $60 \%$ were anticoagulated for 2 weeks to 6 months with only 2 patients 
suffered from complications. However, the severity and sites of thrombosis were not specified. ${ }^{[3]}$ Some authors suggest to anticoagulate high-risk patients, in particular, those who had extensive thrombosis or cerebral sinus thrombosis. There was also a concern for surgical operation and prothrombotic workup before starting anticoagulation. However, high-quality studies on Lemierre's syndrome were limited due to its uncommon nature.

Surgical drainage of sizable and accessible abscess might be beneficial to allow better control of infection. ${ }^{[3,8]}$ As most of the cases can be treated successfully by antibiotics, ligation of the internal jugular vein is seldom needed. Detailed dental assessment and teeth extractions may be required to prevent further septic embolism.

Lemierre's syndrome is the septic embolic complication of recent pharyngeal illness. Fusobacterium spp. accounts for the majority of cases. A high index of suspicion is needed and prolonged antibiotic is advised. It is seldom seen in intensive care but should never be forgotten.

\section{Declaration of patient consent}

The authors certify that they have obtained all appropriate patient consent forms. In the form the patient(s) has/have given his/her/their consent for his/her/their images and other clinical information to be reported in the journal. The patients understand that their names and initials will not be published and due efforts will be made to conceal their identity, but anonymity cannot be guaranteed.

\section{Financial support and sponsorship}

Nil.

\section{Conflicts of interest}

There are no conflicts of interest.

\section{RefEREnCES}

1. Kuppalli K, Livorsi D, Talati NJ, Osborn M. Lemierre's syndrome due to Fusobacterium necrophorum. Lancet Infect Dis 2012;12:808-15.

2. Karkos PD, Asrani S, Karkos CD, Leong SC, Theochari EG, Alexopoulou TD, et al. Lemierre's syndrome: A systematic review. Laryngoscope 2009;119:1552-9.

3. Johannesen KM, Bodtger U. Lemierre's syndrome: Current perspectives on diagnosis and management. Infect Drug Resist 2016;9:221-7.

4. Takano Y, Fukuda K, Takayasu H, Shinmura K, Koizumi G, Sasai M, et al. Liver abscessation and multiple septic pulmonary emboli associated with Lemierre's syndrome: A case report. BMC Res Notes 2015;8:65.

5. Suzuki K, Hayashi Y, Otsuka H, Orita M, Kuwana T, Hashimoto K, et al. Case report; A case of Lemierre's syndrome associated with necrotizing fasciitis and septic embolization. Nihon Naika Gakkai Zasshi 2016;105:99-104.

6. Chamseddin KH, Kirkwood ML. Lemierre's syndrome associated mycotic aneurysm of the external carotid artery with primary internal carotid artery occlusion in a previously healthy 18 -year-old female. Ann Vasc Surg 2016;36:291.e11-291.e. 14.

7. Holm K, Bank S, Nielsen H, Kristensen LH, Prag J, Jensen A, et al. The role of Fusobacterium necrophorum in pharyngotonsillitis - A review. Anaerobe 2016;42:89-97.

8. Riordan T. Human infection with Fusobacterium necrophorum (Necrobacillosis), with a focus on Lemierre's syndrome. Clin Microbiol Rev 2007;20:622-59.

9. Cupit-Link MC, Nageswara Rao A, Warad DM, Rodriguez V. Lemierre syndrome: A Retrospective study of the role of anticoagulation and thrombosis outcomes. Acta Haematol 2017;137:59-65.

10. Rebelo J, Nayan S, Choong K, Fulford M, Chan A, Sommer DD, et al. To anticoagulate? Controversy in the management of thrombotic complications of head \& neck infections. Int J Pediatr Otorhinolaryngol 2016;88:129-35 